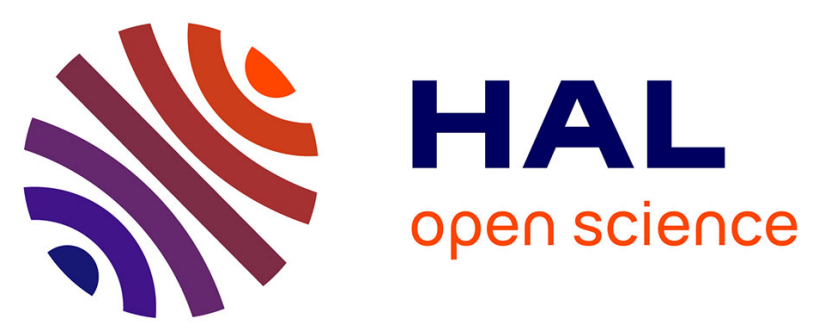

\title{
MODĖLE ANALYTIQUE DU COMPORTEMENT HYDRODYNAMIQUE JUSQU'À RUPTURE PAR FLUAGE UNIAXIAL AVEC DENSIFICATION DU MATÉRIAU
}

Cong Tu Nguyen

\section{To cite this version:}

Cong Tu Nguyen. MODÈLE ANALYTIQUE DU COMPORTEMENT HYDRODYNAMIQUE JUSQU'À RUPTURE PAR FLUAGE UNIAXIAL AVEC DENSIFICATION DU MATÉRIAU. Journal de Physique IV Proceedings, 1991, 01 (C3), pp.C3-887-C3-892. 10.1051/jp4:19913124 . jpa00249929

\section{HAL Id: jpa-00249929 \\ https://hal.science/jpa-00249929}

Submitted on 1 Jan 1991

HAL is a multi-disciplinary open access archive for the deposit and dissemination of scientific research documents, whether they are published or not. The documents may come from teaching and research institutions in France or abroad, or from public or private research centers.
L'archive ouverte pluridisciplinaire HAL, est destinée au dépôt et à la diffusion de documents scientifiques de niveau recherche, publiés ou non, émanant des établissements d'enseignement et de recherche français ou étrangers, des laboratoires publics ou privés. 
Colloque C3, suppl, au Journal de Physique III, Vol. 1, octobre 1991

\title{
MODĖLE ANALYTIQUE DU COMPORTEMENT HYDRODYNAMIQUE JUSQU'À RUPTURE PAR FLUAGE UNIAXIAL AVEC DENSIFICATION DU MATERIAU
}

\section{C.H. NGUYEN}

Groupement de 1'Armement, Services spécialisés et recherche, FS 936, Feuerwerkerstrasse 39, CH-3602 Thoune, Suisse

\begin{abstract}
This model results through an exponential constitutive law in a strong increase of the stress as a function of the strain, up to a value at rupture which depends on a ductility factor of the material. It is applied to five pure metals, including copper in case of shaped charge jet material.

Résumé - Ce modèle aboutit à une augmentation forte, dérivée d'une loi exponentielle de la contrainte en fonction de la déformation, jusqu'à une valeur de rupture dépendant d'un facteur de ductilité du matériau. Il est appliqué à cinq métaux purs, dont le cuivre comme matériau de jet de charge creuse.
\end{abstract}

\section{1.- Introduction}

Le comportement hydrodynamique d'un matériau correspondrait à un domaine de sollicitation à très haute vitesse de déformation ( de l'ordre de $10^{5} \mathrm{~s}^{-1}$ ), dans lequel les contraintes atteintes dépassent de beaucoup celles du domaine élas to-plastique conventionnel, où les déformations également peuvent être beaucoup plus importantes, et surtout où la compressibilité du matériau jouerait un rôle essentiel $/ 1 /$. A défaut d'une caractérisation expérimentale encore inabordable de ce comportement hydrodynamique, un modèle analytique, avec approche de type mécanique, est étudié.

\section{2.- Hodèle de fluage uniaxial avec densification}

Avec l'hypothèse de Bernouilli pour une déformation uniaxiale avec section restant plane, le matériau sollicité hydrodynamiquement peut être simulé par un élément cylindrique ( figure 1 ) s'allongeant par fluage à la contrainte d'écoulement plastique, de valeur usuellement adoptée en dynamique, sous l'effet d'une force de traction croissante au fur et à mesure de l'écrouissage du matériau, en même temps que son volume diminue par densification croissante de celui-ci. Le matériau est supposé a priori comme pouvant être indéfiniment écrouissable et compressible.

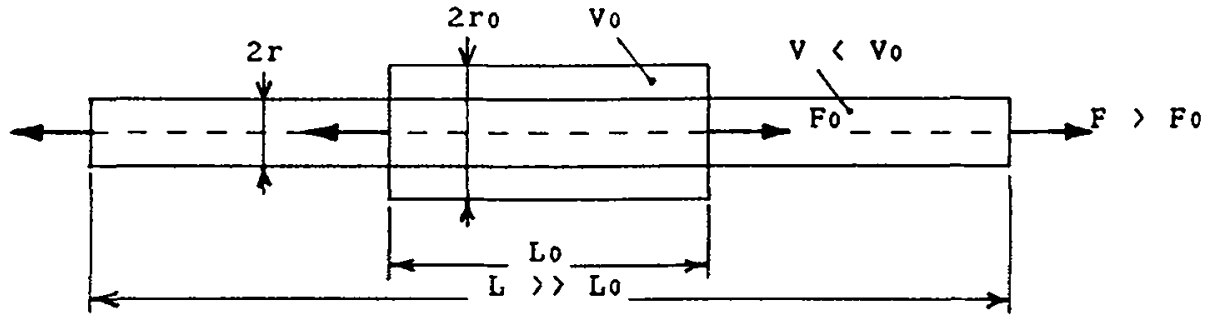

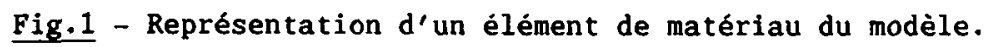


Avec l'hypothèse de la section plane, on néglige les facteurs de second ordre et, en admettant le concept connu que la déformation à très haute vitesse ou pression $n$ 'est plus thermiquement activée ( pas d'adoucissement thermique), une loi de comportement de ce matériau sollicité hydrodynamiquement serait exprimée uniquement par cette variation simultanée de la force de traction sur l'élément et du volume de celui-ci. Cette loi serait valable jusqu'à rupture de l'élément, donc ne devrait normalement être applicable qu'à un comportement en traction.

L'expression analytique du modèle de comportement hydrodynamique étudié se trouverait inscrite dans un domaine de variation, jusqu'à rupture, de la contrainte de fluage en dynamique $\sigma_{f 1}$ en fonction de la déformation $\varepsilon$ ( figure 2), qui est cerné par deux conditions límites dépendant d'un taux de renforcement $\gamma$ du matériau par un effet combiné d'écrouissage ( au sens mécanique du terme) et de densification:

$$
\gamma_{(\varepsilon)}=\alpha_{(\varepsilon)} / \beta_{(\varepsilon)}
$$

où $\alpha$ et $\beta$ désignent respectivement les taux d'écrouissage et de densification du matériau en fonction de la déformation $\varepsilon$, défínis par la combinaison des relations

$$
\begin{cases}F_{(\varepsilon)}=\alpha_{(\varepsilon)} \cdot F_{0} & \text { fonction croissante } \\ V_{(\varepsilon)}=\beta_{(\varepsilon)} \cdot V_{0} & \text { fonction décroissante }\end{cases}
$$

où $F_{0}$ et $V_{0} d^{\prime} u n e$ part et $F_{(c)}$ et $V_{(c)} d^{\prime}$ autre part sont respectivement les valeurs au début du régime hydrodynamique et celles intantanées, en fonction de la déformation $\varepsilon$, de la force appliquée $F$ sur l'élément de matériau ( figure 1 ) et du volume $V$ de celui-ci.

En introduisant la contrainte de fluage $\sigma_{\varepsilon l}$, les relations (2) deviennent

$$
\left\{\begin{array}{l}
\sigma_{f \perp(\varepsilon)} \cdot\left\|r^{2}(\varepsilon)=\alpha_{(\varepsilon)} \cdot \sigma_{f 10} \cdot\right\| r_{0}^{2} \\
\pi r^{2}(\varepsilon) \cdot L_{(\varepsilon)}=\beta_{(\varepsilon)} \cdot \| r_{0}^{2} \cdot L_{0}
\end{array}\right.
$$

ce qui donne, avec $\varepsilon=\left(L / L_{0}\right)-1$, la loi de comportement globale du modèle proposé

$$
\sigma_{\mathrm{f} 1}=\sigma_{\mathrm{f} 10} \cdot \gamma_{(\varepsilon)} \cdot(\varepsilon+1)
$$

où $\sigma_{f 10}$ désigne la contrainte de fluage au début du régime hydrodynamique, dont la valeur peut être estimée à partir de celles plus classiques et expérimentalement connues pour les régimes dynamiques à plus basse vitesse de déformation. Les deux conditions limites de cette loi de comportement sont (figure 2 ) :

\#1: Conditions de "plasticité idéale", où le matériau rend toute l'énergie absorbée en déformation plastique pure, c'est à dire sans travail de renforcement supplémentaire, ni par effet $d^{\prime}$ écrouissage ni par densification, soit avec une variation de contrainte à charge et volume constants, ce qui donnerait

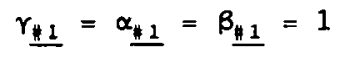

et la loi d'augmentation linéaire de la contrainte de fluage en dynamique

$$
\sigma_{\mathrm{E} 11}=\sigma_{110} \cdot(\varepsilon+1)
$$

\#2: Conditions d'"élasticité limite", au contraire, où le matériau s'oppose au mieux à la déformation plastique, en augmentant au maximum sa résistance par effet combiné d'écrouissage et de densification. Un taux de renforcement limite correspondant du matériau serait fixé par le critère connu définissant la striction à charge maximale d'un diagramme charge-allongemenmt (en relation d'ailleurs avec la notion de vitesse des ondes plastiques dans le matériau): 


$$
\partial \sigma_{\{1(\varepsilon)} / \partial \varepsilon=\sigma_{\ell 1(\varepsilon)}
$$

ce qui donne, en tenant compte de la relation (4) pour isoler $r$

$$
\begin{aligned}
& \quad\left(\partial \gamma_{(\varepsilon)} / \partial \varepsilon\right) \cdot(\varepsilon+1)+\gamma_{(\varepsilon)}=r_{(\varepsilon)} \cdot(\varepsilon+1) \\
& \text { soit } \ln r_{(\varepsilon)}=\int(\varepsilon /(\varepsilon+1)) \cdot d \varepsilon
\end{aligned}
$$

dont l'intégration ( par parties, avec $C t e=-1$ pour $\varepsilon=0$ et $\gamma=1$ ) aboutit à

$$
r_{(\varepsilon) \underline{2}}=e^{\varepsilon /(\varepsilon+1)}
$$

soit à une loi d'augmentation exponentielle de la contrainte hydrodynamique

$$
\sigma_{\mathrm{F} 112}=\sigma_{\mathrm{E} 10} \cdot \mathrm{e}^{\varepsilon}
$$

Entre ces deux lois limites (6) et (11) du modèle de comportement hydrodynamique, une expression analytique peut être trouvée, en première approximation, comme leur moyenne sur la déformation, à même niveau instantané de contrainte (figure 2 ):

$$
\varepsilon=0,5 \cdot\left(\sigma^{*}-1+\ln \sigma^{*}\right) \quad \text { avec } \sigma^{*}=\sigma_{\varepsilon 1} / \sigma_{\varepsilon 10}
$$

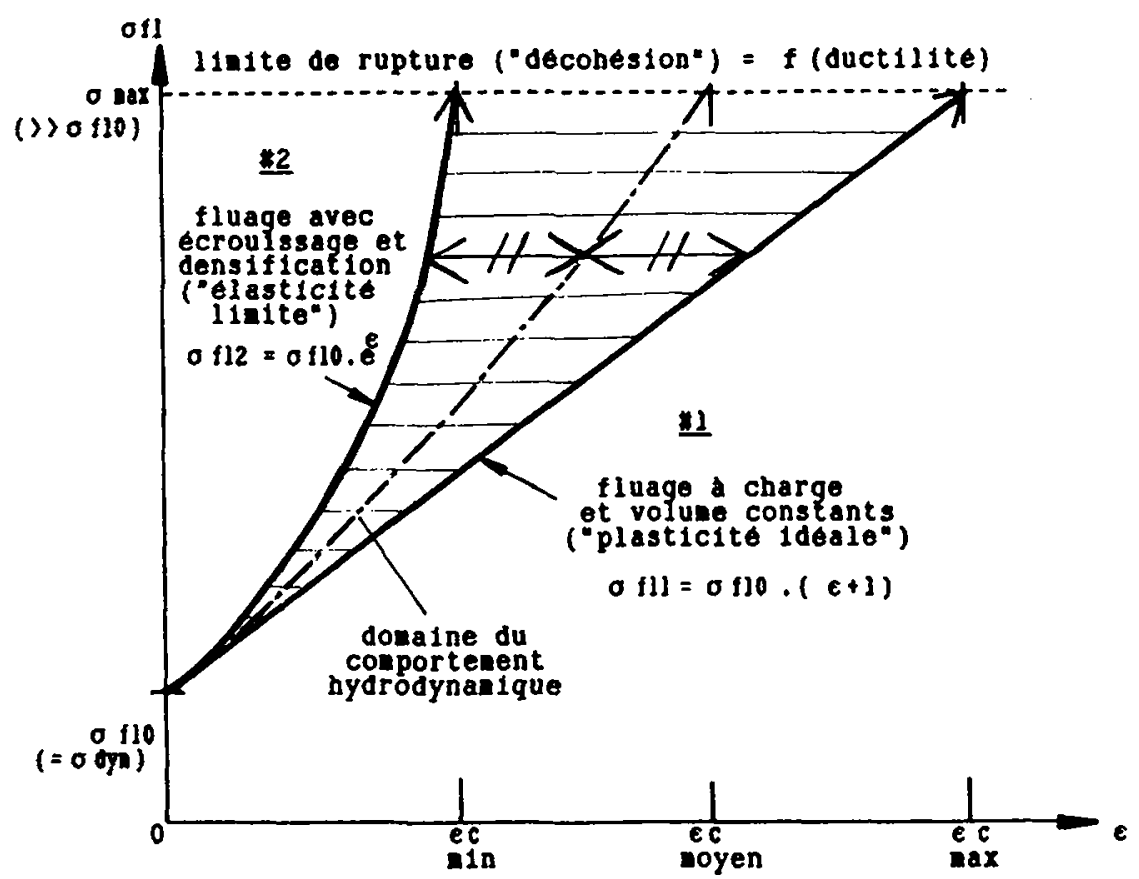

Fig.2 - Courbes limites du modèle de fluage avec densification, délimitant le domaine du comportement hydrodynamique.

Une variation moyenne correspondante du taux de renforcement $\gamma$ peut alors en être déduite. Quant à celles définies sous (1) des taux d'écrouissage $\alpha$ et de densification $\beta$, elles seraient calculées comme des moyennes en contribution à $\gamma$, entre les paires "tout en écrouissage $(\alpha=\gamma ; \beta=1)$ " et "tout en densification $(\alpha=1 ; \beta=1 / \gamma)$ ":

$$
\left\{\begin{array}{l}
\alpha=0,5 \cdot(1+\gamma) \\
\beta=0,5 \cdot(1+(1 / \gamma))
\end{array}\right.
$$




\section{3.- Caractéristiques à la rupture du nodèle}

Ce modèle analytique proposé pour le régime hydrodynamique serait valable jusqu'à la rupture du matériau, correspondant par définition à la valeur maximale possible de résistance en dynamique. Celle-ci serait ici déterminée d'après les critères en mécanique de rupture, sur la base des cercles de Mohr extrapolés des états de contraintes limites, respectivement de contrainte plane, de déformation plane et également de contrainte triaxiale (figure 3 ). L'extrapolation serait valable avec l'hypothèse d'un fluage uniaxial intense et à grande vi tesse ( phénomène transitoire). En fonction de la ductilité du matériau et dans les cas normaux de rupture, le taux de croissance de cette résistance maximale rapportée à la limite de fluage en dynamique - facteur $\sigma^{*}$ à la relation (12) - varierait entre une valeur minimale, pour l'état de déformation plane, qui est calculée ( avec Tresca ou von Mises $\sigma_{\mathrm{f} 10}=\sigma_{\mathrm{E} 1},(1-2 \mathrm{U})$ où le coefficient de Poisson $v=0,3$ pour les métaux )

$$
\sigma_{E I \text { min }}=2,5 . \sigma_{f 10}
$$

et qui, portée dans (12), correspond à un taux de déformation critique de $120 \%$, et une valeur maximale, pour l'état de contrainte plane, qui est estimée égale à

$$
\sigma_{\ell 1 \underline{\max }}=8 . \sigma_{t 10}
$$

pour correspondre à un taux de déformation critique de $450 \%$, ceci à la suite de calculs, vérifiés par expériences de tir, de la fragmentation optimale d'un jet de charge creuse en cuivre ( après un allongement maximal observé de $550 \%$ ) $/ 2 \%$. Les paramètres de ductilité considérés peuvent être la maille cristalline, avec éventuel changement de phase, la microstructure, ou encore une fabrication par P/M...

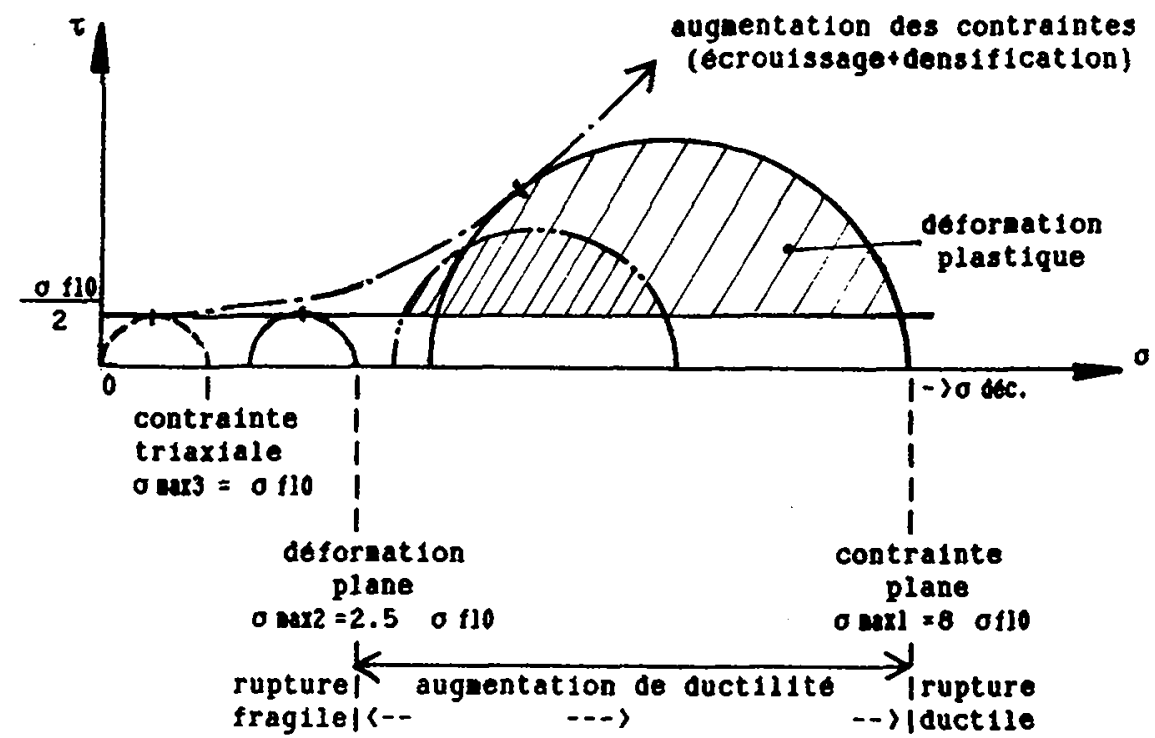

Fig.3 - Cercles de Mohr des états de contraintes limites du modèle, pour l'estimation de la limite de rupture en hydrodynamique en fonction de la ductilité du matériau.

\section{4.- Application aux cas de quelques métaux purs}

Les calculs de la loi de comportement hydrodynamique d'après (12) ont été effectués pour quelques métaux purs avec différentes mailles cristallines ( figure 4 ). Les valeurs de la contrainte de fluage en dynamique $\sigma_{f 10}$ ont été estimées à partir 

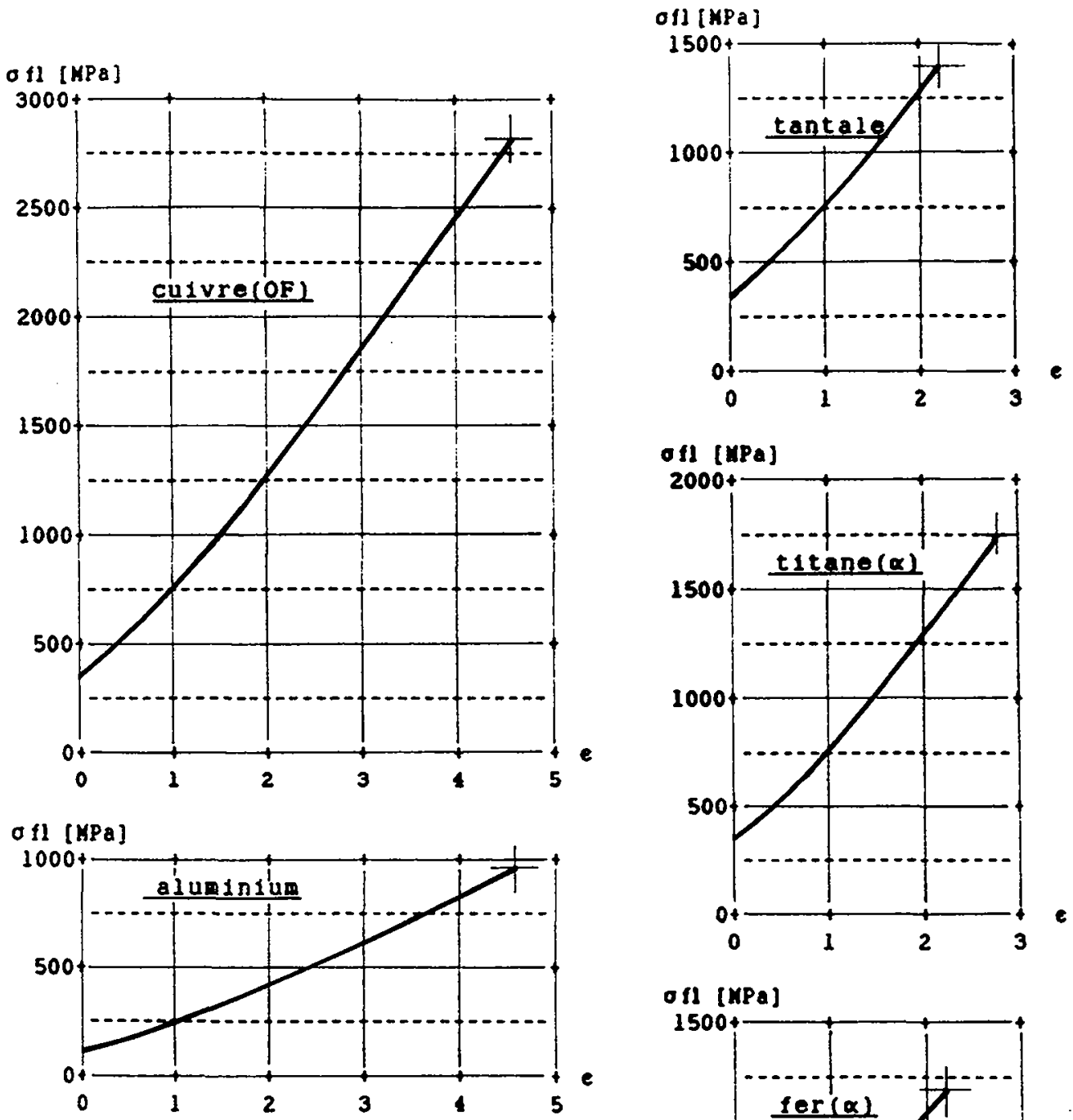

\begin{tabular}{|c|c|c|r|r|r|}
\cline { 2 - 6 } \multicolumn{1}{c|}{} & Cu & Al & Ta & T1 & Fo \\
\hline$\sigma 110[\mathrm{MPa}]$ & 350 & 120 & 350 & 350 & 300 \\
\hline O $14 \times[\mathrm{MPB}]$ & 2800 & 960 & 1400 & 1750 & 1200 \\
\hline ec l-] & 1.5 & 4.5 & 2.2 & 2.8 & 2.2 \\
\hline
\end{tabular}

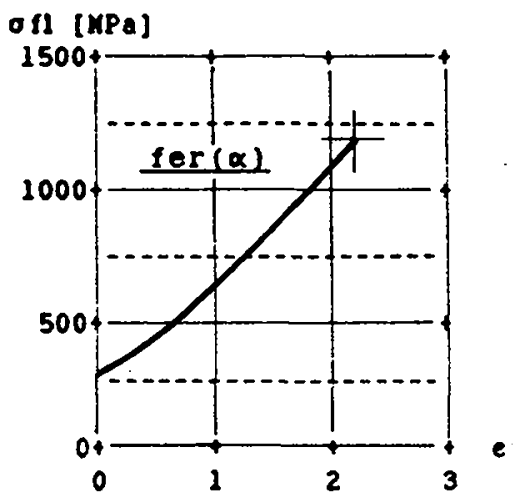

Fig. 4 - Courbes contrainte-déformation calculées pour quelques métaux purs d'après le modèle du comportement hydrodynamique, avec tableau correspondant des principales caractéristiques. 
de la littérature: cuivre (qualité $O F$ ) $13,4,5,6 /$, aluminium $13,4,5 /$, tantale $/ 3 /$, titane (phase $\alpha$ ) $14,5 /$ et fer (phase $\alpha$ ) $15,6 /$. Les valeurs de la contrainte à la rupture $\sigma_{\max }$, donc celles du taux de déformation critique $\varepsilon_{c}$, ont été calculées, tenant compte des relations (14) et (15), avec un facteur $\sigma^{*}$ égal à 8 (=max) pour la maille $\langle f c c\rangle$, à 5 pour la maille $\langle$ hex $\rangle$ et à 4 pour la maille $\langle b c c\rangle$. Un éventuel changement de phase sous choc serait une discontinuité sortant du cadre du modèle.

\section{5.- Discussion des résultats - Cas de la charge creuse}

- Coefficients de viscosité dynamique - Une appréciation de ces valeurs calculées avec le modèle hydrodynamique ici présenté peut être faite en reportant cellesci dans un autre modèle connu, celui du régime visco-plastique, qui s'écrit par rapport au régime en statique $\sigma_{s t a t}$ ( limite d'élasticité ) et proportionnellement à la vitesse de déformation $\varepsilon^{\prime}$ ( au facteur du coefficient de viscosité dynamique $\mu$ du matériau près, lequel varierait selon $\left.\varepsilon^{\prime}\right) / 7 /$ :

$$
\sigma_{\text {dyn }}=\sigma_{\text {stat }}+\mu_{\left(\varepsilon^{\prime}\right.} \cdot \varepsilon^{\prime}
$$

Avec les valeurs pour $\varepsilon^{\prime}$ de $10^{4}$ et $10^{6} \mathrm{~s}^{-1}$ respectivement au début et à la fin du régime hydrodynamique, on obtient en effet des valeurs cohérentes du coefficient de viscosité dynamique $\mu$, pour les exemples à la figure 4, passant d'environ 1 à $2.10^{4} \mathrm{~Pa} . \mathrm{s}$ pour le début jusqu'à environ 1 à $3.10^{3} \mathrm{~Pa} . \mathrm{s}$ pour la fín.

- Equations d'état - En portant les valeurs calculées par (13) du taux de densification $\beta$ à la rupture pour ces métaux, qui seraient comprises entre 0,85 et 0,9 , dans leurs équations d'état, il est encore possible d'avoir une vérification par le niveau de la pression atteinte. Pour le cuivre, la pression calculée serait de 350-400 kbar $/ 8 \%$, correspondant donc au cas du collapse d'une charge creuse.

- Recristallisation dynamique - Avec les valeurs calculées des taux de déformation critique $\varepsilon_{c}$ ( figure 4 ), on peut en déduire les montées adiabatiques correspondantes en température. Toujours dans l'exemple du cuivre, elle serait d'environ $500{ }^{\circ} \mathrm{C} / 9 /$, donc également plausible à l'état d'un jet de charge creuse. Cette température d'environ la moitié de celle de fusion du matériau justifierait son état de recristallisation dynamique ( ayant lieu en même temps que la déformation à haute vitesse), dont la structure sub-micronique a été observée $/ 10 /$.

- Mode de rupture "par effilochage" - Ce régime, de très grande déformation à très grande vitesse, s'expliquerait par un processus de striction quasi-instantanée, soit avec des paliers de glissement multiples et larges sur toute la section de l'élément de matériau. La rupture finale se ferait par un mode donnant lieu à un matériau restant qui serait sous forme de couches superposées, séparées par des fissures orientées selon l'axe de traction, comme il a été effectivement observé sur des fragments récupérés de jets de charge creuse $/ 10 \%$. Ce mode de rupture ne ressemble ainsi pas à ceux déjà répertoriés en dynamique des matériaux $/ 11,12 /$.

\section{Références}

/1/ BRONIAREK, C.A., Tuskegee Inst., AL, Rep. AR0-18447.1-EG-H (1982).

/2/ NGUYEN, C.H., Eidg. Munitionsfabrik Thun, Bericht X 010161 (1984).

/3/ WILKINS, M.L. et al, J. Appl. Phys. 44, 3 (1973) 1200.

14/ TSAO, M.C. et al, Oxford Univ., Tech. Rep. AFML-TR-73-177 (1973).

15/ DUFFY, J., Wright-Pat terson AF Base, Int. Rep. AFWAL-TR-82-4024 (1982).

/6/ JOHNSON, G.R. and COOK, W.H., 7th Int. Symp. Ballist., The Hague (1983) 541.

17/ WALTERS, W.P., Ballistic Research Lab., Mem. Rep. ARBRL-MR-02941 (1979).

/8/ WALSH, J.M. et al, Phys. Rev. 108, 2 (1957) 196.

/9/ CHOKSHI, A and MEYERS, M., Scr. METALL. MAT. 24 (1990) 605.

/10/ NGUYEN, C.H., Eidg. Munitionsfabrik Thun, Bericht X 010322 (1987).

/11/ SEAMAN, L. et al, SRI, Tech. Rep. AFWL-TR-71-156 (1972).

/12/ DORMEVAL, R., Proc. IMPACT'87, Bremen, DGM Inf.Ges. (1988) 43. 